\title{
A difficult case of robotic splenic-flexure mobilization, performed by a trainee surgeon with a dual Davinci console
}

\author{
T. Petropoulou' · S. Amin ${ }^{1}$
}

Received: 30 December 2019 / Accepted: 7 April 2020 / Published online: 27 April 2020

(c) The Author(s) 2020

Robotic technology is growing up fast and there is a need for proper training and safe implementation of the technique.

The da Vinci robot is unique because among other features, it also comes with a dual console, which can help in fast and safe training of surgeons. The proctor can watch the trainee surgeon and point out to them the right plane of dissection, or draw the right plane on the screen. They can also take over one or more of the instruments, if needed. One of the most difficult steps of a robotic anterior resection is the splenic flexure mobilization.

In this video, we present our standardized technique in the case of difficult splenic flexure mobilization, performed by a trainee robotic surgeon, with the guidance of the proctor on a dual console.

We use a fully robotic single, side-docking technique, which means we do not have to dock the robot twice for splenic-flexure mobilization.

We always start by skeletonizing and dividing the inferior mesenteric artery near its origin with the aorta. We then continue in a medial-to-lateral fashion on the infracolic, suprapancreatic plane. We carry on the dissection cranially in this plane until we enter the lesser sac and see the back wall of the stomach. We then divide the gastrocolic omentum.

We routinely take down the splenic flexure, in all our anterior resections, because we believe that it gives the extra length needed for a tension-free anastomosis.

Author contributions We describe how the dual console can contribute to training of surgeons and the results that can be obtained from an advanced robotic fellowship. Moreover, this is our standardized technique, which can fully achieve mobilization of the splenic flexure without the need for dual docking.

Funding No funding was used for the study.

Data availability The datasets used and/or analyzed during the current study are available from the corresponding author on a reasonable request.

\section{Compliance with ethical standards}

Conflict of interest There are no financial or non-financial competing interests to declare.

Ethics approval This video does not contain personal identifiable data; however, patients were aware and in agreement for their cases to be recorded and published.

Informed consent Informed consent was obtained from all patients.

Open Access This article is licensed under a Creative Commons Attribution 4.0 International License, which permits use, sharing, adaptation, distribution and reproduction in any medium or format, as long as you give appropriate credit to the original author(s) and the source, provide a link to the Creative Commons licence, and indicate if changes were made. The images or other third party material in this article are included in the article's Creative Commons licence, unless indicated otherwise in a credit line to the material. If material is not included in the article's Creative Commons licence and your intended use is not permitted by statutory regulation or exceeds the permitted use, you will need to obtain permission directly from the copyright holder. To view a copy of this licence, visit http://creativecommons.org/licenses/by/4.0/.

Publisher's Note Springer Nature remains neutral with regard to jurisdictional claims in published maps and institutional affiliations.

Electronic supplementary material The online version of this article (https://doi.org/10.1007/s10151-020-02216-7) contains supplementary material, which is available to authorized users.

T. Petropoulou

thalia_pet@hotmail.com

1 Department of Colon and Rectal Surgery, Sheffield Teaching Hospitals NHS, Sheffield, UK 